\title{
Breve comentario sobre EL PAPEL DEL MÉTODO INVERSO DE TANGENTES EN LA CONTROVERSIA SOBRE EL CÁLCULO INFINITESIMAL
}

\author{
Miguel Palomo. Universidad de Sevilla
}

\begin{abstract}
Resumen: Aunque el origen de la controversia por la invención del cálculo infinitesimal se suele situar en 1699 cuando Fatio de Duillier publica su "Lineae brevissimi descensus investigatio geometrica duplex", probablemente el origen se encuentra en el intercambio de métodos relativo al método inverso de tangentes que mantuvieron Fatio de Duillier y
\end{abstract} Leibniz entre 1690 y 1692.

Palabras clave: Leibniz, Fatio, Método inverso de las tangentes.

Brief commentary on the role of the inverse method of tangentes in the controversy on the infinitesimal calculation

Abstract: It is said the controversy over the invention of the calculus started in 1699 with the publication of "Lineae brevissimi descensus investigatio geometrica duplex" by Fatio de Duillier. The aim of this paper is to establish that probably the controversy started with an interchange of methods between Leibniz and Fatio de Duillier in 1691-1692 regarding the inverse method of tangents.

Keywords: Leibniz, Fatio, inverse method of tangents.

Recibido: 20/06/2016 Aprobado: 29/08/2016 
Miguel Palomo

\section{Introducción}

La controversia sobre la invención del cálculo infinitesimal ha sido documentada en multitud de libros y artículos especializados. Esto se explica por la relevancia que tuvo la invención del cálculo para el uso las ciencias, ya que supuso un enorme avance en matemáticas que tuvo una rápida influencia en trabajos de física y geometría. Pero además, también se explica por la singularidad del caso, ya que supuso una auténtica lucha de titanes entre Newton y Leibniz, así como entre la ciencia hecha en las islas británicas y aquella realizada en la Europa continental.

Respecto al origen exacto de la controversia, sin embargo, y a pesar de la gran cantidad de obras dedicadas a la llamada "guerra del cálculo", hay todavía detalles que se encuentran sin aclarar y que pueden ocasionar un cambio de interpretación.

Aunque en casi todos los casos se da por hecho que el origen de la controversia se encuentra en un artículo escrito en 1699 por Fatio de Duillier, hay que retrotraerse hasta 1690 para encontrar el origen de estas discusiones las cuales, a la larga, ocasionarían un declive de Leibniz en su vida tanto pública como privada. Por ello, es de especial relevancia conocer el momento exacto en el que la controversia tiene lugar, para así poder analizar cómo la última etapa de una de las mentes más brillantes de la historia pudo estar determinada no por sus aportaciones científicas sino por las pasiones que dominan al género humano. 
Controversia sobre el cálculo infinitesimal

\section{La bibliografia especializada respecto al origen de la controversia}

La bibliografía especializada da por hecho que la controversia comienza cuando Fatio de Duillier, antiguo asistente primero de Christiaan Huygens y más adelante de Newton, da la voz de alarma en su escrito "Lineae brevissimi descensus investigatio geometrica duplex" (Doble investigación geométrica de la línea descendente más breve), un texto donde Fatio analiza la curva braquistócrona, publicado con el beneplácito de la Royal Society en 1699 y donde, además, implícitamente afirma que Leibniz ha tomado prestado el cálculo de Newton ${ }^{1}$.

Así lo estiman la gran mayoría de investigadores en la materia, como por ejemplo Knobloch ${ }^{2}$, Echevarría ${ }^{3}$ o Rupert Hall, quien dice que, antes del artículo de Fatio, no había indicio alguno de polémica en este asunto entre británicos y continentales y que existía una ignorancia natural entre los orígenes tanto en unos como en otros ${ }^{4}$. Siguiendo esta estela, Javier de Lorenzo sitúa el origen de la controversia a finales de siglo después de las acusaciones

${ }^{1}$ F. de Duillier, Lineae brevissimi descensus investigatio geometrica duplex, Londres 1699: 18.

${ }^{2}$ E. Knobloch, "Leibniz and the Brachistochrone", Documenta Mathematica. Extra Volume ISMP, 2012: 18.

${ }^{3}$ J. Echevarría, "Valores contrapuestos en la controversia Newton-Leibniz" en: A. J. Durán y J. Ferreirós (eds.) Matemáticas y matemáticos, Sevilla: Secretariado de Publicaciones de la Universidad de Sevilla, 2004: 87.

${ }^{4}$ A. Rupert Hall, Philosophers at War. The Quarrel Between Newton and Leibniz, Cambridge: Cambridge University Press, 1980: 118. 
de los partidarios de Newton ${ }^{5}$, así como José Babini, quien afirma que el primer ataque directo lo realiza Fatio en $1699^{6}$. Socrates, investigador que ha dedicado una monografía a la controversia sobre el cálculo, solamente afirma que Huygens intentó promover una correspondencia personal entre Leibniz y Fatio $^{7}$ y da por sentado que el origen de la controversia se encuentra en $1699^{8}$. Mary Sol de Mora, editora de los escritos matemáticos de la edición de las obras en castellano de Leibniz, afirma que la relación entre Fatio y Leibniz era agria ya antes de finales de siglo ${ }^{9}$ y habla de que la polémica no estalla hasta $1699^{10}$.

Esta es la tónica general que nos encontramos en las obras dedicadas a los orígenes del cálculo desde que comenzaron a realizarse análisis objetivos de la controversia, mientras que otros no especifican un momento concreto. En De Morgan ${ }^{11}$, nada se nombra sobre el intercambio entre Fatio y Leibniz. Tampoco lo hace Boyer en su historia del cálculo ${ }^{12}$. Por su parte, Baron ${ }^{13}$

5 J. de Lorenzo, "Estudio preliminar" en: G. W. Leibniz, Análisis infinitesimal, Madrid: Tecnos, 1987: LXXIII.

${ }^{6} \mathrm{~J}$. Babini, El cálculo infinitesimal: origen-polémica, Buenos Aires: Editorial universitaria de Buenos Aires: 59.

${ }^{7}$ J. Socrates, The Calculus War: Newton, Leibniz, and the Greatest Mathematical Clash of All Time, Harpenden: High Stakes, 2009: 141.

${ }^{8}$ Íbid., 175-176.

${ }^{9}$ G. W. Leibniz, Obras fillosóficas y cientificas, Granada: Comares, vol.7A: 341.

${ }^{10}$ Íbid., vol.7A: 355.

${ }^{11}$ A. De Morgan, Essays on the Life and Work of Newton, Chicago: Open Court, 1914.

${ }^{12}$ C. B. Boyer, The History of the Calculus and its Conceptual Development, New York: Dover Publications, 1959. 
señala el método inverso de tangentes como un punto importante en el desarrollo del cálculo infinitesimal por parte de Leibniz, aunque sin mencionar el papel que juega en el origen de la controversia y sin mencionar a Fatio de Duillier. Por último, O’Hara y Wahl, editores del volumen 8 de la edición de las obras completas de Leibniz ${ }^{14}$, donde se encuentran publicadas las cartas entre Leibniz y Fatio, aunque afirman que ambos tuvieron un intercambio de métodos entre 1691 y 1692, no hacen mayor referencia a la importancia de este hecho, centrándose más bien en la polémica de 1699.

Sin embargo, sí hay quienes han señalado que el origen se encuentra más atrás en el tiempo. Westfall afirma que fue en 1699 cuando Fatio aviva las llamas de la controversia ${ }^{15}$. Tanto esa afirmación como el resto de su recapitulación de la controversia da a entender que ésta se retrotrae a los años anteriores a 1699, llegando incluso a hablar de la idea de Newton de formalizar en un artículo alrededor de 1690 su método de fluxiones para ser publicado ${ }^{16}$, pero también de la carta de Leibniz a Hans Sloane, secretario de la Royal Society, en 1711 como inauguradora de "una acalorada controversia sobre las afirmaciones de prioridad en la invención del cálculo" ${ }^{17}$. Westfall,

${ }^{13}$ E. Baron, The Origins of the Infinitesimal Calculus, Cambridge: Cambridge University Press, 1969.

${ }^{14}$ G.W. Leibniz, Sämtliche Schriften und Briefe, Deutschen Akademieder Wissenschaften zu Berlin (ed.), Berlín: Darmstadt, 1923ss.

${ }^{15}$ R. S. Westfall, Never at rest, a biography of Isaac Newton, Cambridge: Cambridge University Press, 2005: 539.

16 Íbid., 512.

17 Íbid., 698. 
sin embargo, no estipula un comienzo claro de la controversia ni hace referencia al intercambio de métodos entre Leibniz y Fatio.

Aiton, biógrafo de Leibniz, afirma que la semilla de la controversia es plantada por Fatio en carta de éste último a Huygens el 28 de diciembre de $1691^{1819}$. Este es, ciertamente, el primer lugar donde Fatio afirma directamente que Leibniz no ha hecho referencia a Newton en los artículos de las Acta Eruditorum donde Leibniz publicó por primera vez, aunque de manera no muy clara, su nuevo cálculo. Antonio J. Durán también hace referencia a esta carta entre Fatio y Huygens, aunque sin mencionar el contexto y origen de ésta y señalando que, en ella, probablemente Fatio estaba simplemente transmitiendo aquello que le oía decir a Newton ${ }^{20}$, algo que también opina Westfall ${ }^{21}$.

Por otro lado, Antognazza también habla de este intercambio de impresiones entre Fatio y Huygens, aunque ella afirma que la semilla del comienzo de la controversia se encuentra en la carta de Fatio a Huygens del 15 de febrero de $1692^{22}$, carta en la que, efectivamente, Fatio se reafirma en su

${ }^{18}$ E. J. Aiton, Leibniz: una biografia, Madrid: Alianza: 237.

${ }^{19} \mathrm{Ch}$. Huygens, Oeuvres Complètes Oeuvres complètes de Christiaan Huygens, D. B. Haan, J. Bosscha, D. J. Korteweg, y J. A. Vollgraff (eds.), La Haya: Martinus Nijhoff, vol. 10, n.2723: 213-215.

20 A. J. Durán, La Polémica sobre la invención del cálculo infinitesimal: escritos y documentos, Barcelona: Crítica, 2006: 94.

${ }^{21}$ Westfall, op. cit., 514.

${ }_{22}^{2}$ M. R. Antognazza, Leibniz: an Intellectual Biography, Cambridge: Cambridge University Press, 2009: 356; Huygens, op. cit., 10, n. 2739: 257-259. 
opinión de que Leibniz ha plagiado el cálculo a Newton, pero que no es la primera en la que nombra el supuesto plagio.

Por último, Alfonso Pérez de Laborda hace un muy buen análisis del origen de la controversia, llegando a estudiar ambas cartas de Fatio a Huygens (las del 28 de diciembre de 1691 y la del 15 de febrero de 1692) ${ }^{23}$, así como brevemente las cartas entre Leibniz y Huygens ${ }^{24}$. Sobre ésta última correspondencia, sin embargo, se centra en el reto de la catenaria propuesto por Bernoulli y la visión de Huygens sobre el cálculo leibniziano, en lugar de hablar del método inverso de tangentes y su relación con Fatio.

Por todo ello, aunque Westfall, Aiton, Durán, Antognazza y Pérez de Laborda han buscado más allá del artículo de Fatio de 1699 a la hora de situar la raíz de la controversia y han retrotraído sus orígenes hasta 1692, las cartas que han señalado como el origen son una reacción de Fatio ante un intercambio fallido de métodos entre Leibniz y él mismo, por lo que el origen de la controversia tiene su comienzo incluso más atrás en el tiempo.

\section{El intercambio de métodos entre Leibniz y Fatio}

Todo comienza en el intercambio de cartas que Leibniz mantiene con Christiaan Huygens. Esta correspondencia es extensa y compleja, abarcando desde 1672 hasta 1695 . Dentro de los diferentes asuntos que Leibniz y su

${ }^{23}$ A. Pérez de Laborda, Leibniz y Nerwton. I: la discusión sobre la invención del cálculo infinitesimal, Salamanca: Universidad Pontificia de Salamanca, 1977:144.

${ }^{24}$ Íbid., 147-150. 
antiguo maestro Huygens discuten en sus cartas, se encuentra una discusión sobre el método inverso de tangentes. Este método ya fue tratado por Leibniz en 1673 en su artículo Methodus tangentium inversa, seu de functionibus. En él Leibniz trataba dos problemas inversos: encontrar la subtangente de una curva partiendo de la relación dada entre las abcisas y las ordenadas; y encontrar la relación entre las abcisas y las ordenadas partiendo de alguna propiedad de la subtangente, de la subnormal o de algún segmento dado ${ }^{25}$.

Para hablar del origen de la controversia del cálculo, debemos avanzar ahora hasta unos años más adelante, en una discusión que comienza en la carta de Huygens del 24 de agosto de $1690^{26}$. En esta carta y con el objetivo de poner a prueba el cálculo infinitesimal de Leibniz, Huygens le anima a buscar una ecuación que exprese la relación entre los segmentos $\mathrm{AB}$ y $\mathrm{BC}$ de un círculo dado, y también a buscar la ecuación cuando BD es $\frac{2 x x y-a a x}{3 a a-2 x y}$, siendo $a$ una línea dada ${ }^{27}$. Leibniz rápidamente se dedicó a responder a estas cuestiones en su carta de mitad de octubre de $1690^{28}$, carta que nunca llegó a enviar, y también en la siguiente ${ }^{29}$. Además, Leibniz envió una segunda elaboración del problema a Huygens en carta del 7 de noviembre ${ }^{30}$.

${ }^{25}$ A. Sierpinska, Understanding in Mathematics, Londres: Falmer, 1994: 99.

${ }^{26}$ G. W. Leibniz, op. cit., III 4, n.271: 545ss.

27 Íbid., III 4, n.271: 548.

${ }^{28}$ Íbid., III 4, n.282: 588-619.

${ }^{29}$ Íbid., III 4, n.283: 619-624.

${ }^{30}$ Íbid., III 4, n.287: 640-647. 
En las cartas posteriores, Huygens envía una segunda curva a Leibniz ${ }^{31}$ y continúan discutiendo sobre el método inverso de tangentes hasta que Huygens recuerda, en la carta del 19 de diciembre de 1690, que más de un año atrás Fatio de Duillier había utilizado un método similar, pero que, por tratarse de un asunto de larga discusión, no lo había estudiado aún ${ }^{32}$. Leibniz responde que le gustaría saber en qué casos el método de Fatio es más sencillo de aplicar ${ }^{33}$, a lo que Huygens afirma que, a pesar de que durante este tiempo Fatio ha seguido desarrollando su método, éste encuentra las mismas dificultades que Huygens proponía y no puede resolver aquellos casos que contienen incógnitas y más de un término: por ejemplo, si la subtangente es $\frac{y y \sqrt{a a-x x}}{a x}$ siendo $x$ la abcisa, $y$ aplicándose a los ángulos rectos y siendo $a$ una línea conocida ${ }^{34}$. Afirma Huygens que si el método de Leibniz no se detiene en estas raíces, entonces es más completo que el de Fatio.

La respuesta de Leibniz puede interpretarse como entusiasta, pues dice que las palabras de Huygens han despertado su interés y apreciación. Señala Leibniz que, de quererlo Fatio, él puede ayudarle en esta investigación sobre las curvas cuyas subtangentes son conocidas, ya que este es de los asuntos más difíciles e importantes en geometría ${ }^{35}$. Esta voluntad de ayudar a Fatio se concentra en la siguiente proposición: que si Fatio le envía su solución

\footnotetext{
31 Íbid., III 4, n.291: 654-655.

32 Íbid., III 4, n.296: 689-690.

${ }^{33}$ Íbid., III 5, n.6: 47.

${ }^{34}$ Íbid., III 5, n.8: 56-57.

${ }^{35}$ Íbid., III 5, n.9: 59.
} 
para las dos curvas propuestas por Huygens, él le enviaría la suya para aquellas donde Fatio ha encontrado dificultad: " $\mathrm{Si}$ a Fatio le parece bien comunicarme su método para vuestras dos líneas, yo le comunicaría el mío para aquellas dos donde de momento ha encontrado dificultad" ${ }^{36}$. Este es un punto importante, pues la discusión culminará en el contenido del método que Leibniz enviará más adelante.

Fatio, en principio, decide que va a intentar resolver estos problemas por su cuenta, aunque Huygens afirma que no deja de decirle que muestre lo que ha encontrado, ya que el problema es de gran utilidad ${ }^{37}$. E1 21 de abril de 1691, sin embargo, Huygens señala que Fatio le ha mostrado todo su método y que ambos lo estudiaban cada día con la intención de mejorarlo ${ }^{38}$, y finalmente afirma que Fatio está dispuesto a aceptar la propuesta de Leibniz según afirma Huygens el 5 de mayo de 1691.

El trato, según Huygens, sería que él enviaría a Leibniz el método de Fatio qui en verité est tres belle, tras recibir Huygens el de Leibniz, a quien le pide que sea claro en su exposición para poder comprender adecuadamente su cálculo diferencial ${ }^{39}$. Nótese que Leibniz pretendía hacer llegar solamente una parte de su método, tal y como he señalado más arriba (solamente lo que él consideraba necesario para que Fatio llegase a la solución), mientras que

36 "Si M. Fatio trouve bon de me communiquer sa methode pour vos deux lignes[,] je luy communiqueray la mienne pour ces deux d'à present, oú il a trouvé de la difficulté" Íbid., III 5, n.9: 60 .

37 Íbid., III 5, n.13: 85.

${ }^{38}$ Íbid., III 5, n.18: 104.

${ }^{39}$ Íbid., III 5, n.21: 111-112. 
Huygens está solicitando el método por completo de Leibniz. El trato, sin embargo, es aceptado por Leibniz en su carta del 27 de mayo de 1691, afirmando que "Cuando haya descansado un poco de las distracciones del viaje que me han impuesto por necesidad las investigaciones en los archivos y bibliotecas, enviaré mi método como intercambio de aquel del Sr. Fatio" ${ }^{40}$. Aquí Leibniz no parece hacer referencia a una parte sino a su método por completo.

Leibniz prepara un artículo en el que presenta su intercambio dirigido a Fatio con el título "Methodus, qua innumerarum Linearum Constructio ex data proprietate Tangentium seu aequatio inter Abscissam et Ordinatam ex dato valore Subtangentialis, exhibetur", enviado a Huygens a través de Meier el 5 de octubre de $1691^{41}$, en el que envía parte de su solución a los lugares donde Fatio tenía problemas.

A pesar de su interés, Leibniz se había demorado unos meses en enviar este artículo, lo cual hacía sospechar a Huygens que Leibniz no pensaba cumplir su parte del trato en su carta del 16 de noviembre de $1691^{42}$. Además, señala que Fatio había dejado La Haya dos meses atrás, camino de Inglaterra, y que se llevó la carta en la que había mostrado el método a Huygens. Afirma también que el método había sido desarrollado en esa carta

\footnotetext{
40 "Quand j'auray respiré un peu des distractions du voyage dont les recherches dans les archives et Bibliotheques m'ont imposé la necessité, j’envoyeray ma methode en echange de celle de M. Facio” Íbid., III 5, n.22: 114.

${ }^{41}$ Íbid., III 5, n.41: 181-189.

42 Íbid., III 5, n.46: 201.
} 
desde su primera versión ${ }^{43}$ y que él sólo tenía los resultados a los dos problemas, por lo que tendría que deducir la regla.

Es el 1 de enero de 1692 cuando, tras estudiar el artículo dirigido a Fatio, Huygens escribe a Leibniz con un tono airado, pues se ha percatado de que Leibniz le ha mandado solamente una parte del método y no el método por completo. Aquí está el punto clave de la cuestión: primero Leibniz propuso mandar su método para aquella parte en la que Fatio tenía problemas ( 2 de marzo de 1691); Fatio acepta el trato y Huygens propone un intercambio de métodos entre ellos dos, sin especificar si se refería al método por completo o solamente una parte, por lo que se entiende que se refiere a ambos métodos completos (5 de mayo de 1691); por último Leibniz afirma que enviará su método a Fatio en una corta carta escrita con prisa según se deduce de sus palabras y su corta extensión (27 de mayo de 1691).

El 8 de enero de 1692 Leibniz responde a Huygens, presumiblemente nada más leer la carta del 1 de enero, diciendo que espera que no le haya enseñado su método a Fatio, que él ha cumplido el trato al enviar su parte del método y que esperaba de Fatio la solución de los problemas utilizando su método. Afirma, por último, que la parte del método que ha enviado tiene el

\footnotetext{
${ }^{43}$ Los manuscritos de Fatio de Duillier se encuentran en la Biblioteca de la Université de Genève, Suiza. Aunque muchos textos de Fatio se encuentran accesibles (por haberlos publicado en revistas académicas de la época) y algunas de sus cartas (como las dirigidas a Huygens y a Newton) han sido editadas, no existe una edición de sus obras completas. Por ello, no he podido acceder a la solución de Fatio, la cual previsiblemente se encuentra entre sus manuscritos.
} 
mismo valor que todo el de Fatio ${ }^{44}$. A pesar de que Leibniz esperaba que Fatio cumpliese su parte, nunca llegó a recibir su método, pues, aunque tras la polémica Huygens propone a Leibniz enviarle el método de Fatio si así lo desea ${ }^{45}$, este gesto de buena fe por parte del holandés es declinado por Leibniz ${ }^{46}$.

Tal y como muestra el caso y como mostrará la deriva que acaba tomando el desarrollo de la polémica más adelante, Leibniz en ningún momento actúa de mala $\mathrm{fe}^{47}$. Se trata, más bien, de una serie de malentendidos (no fue el único que tuvo con Huygens) que en este caso implica a una tercera persona, Fatio, quien como se puede comprobar en sus cartas con Huygens, reacciona de un modo excesivamente personal a un fallido intercambio académico.

\section{Las cartas entre Fatio y Huygens}

Las cartas entre Fatio y Huygens nos ofrecen una visión importante sobre este origen de la controversia, pues confirman que la causa de esta controversia y de su levantamiento por parte de Fatio está ocasionado por el fallido intercambio sobre el método inverso de tangentes. Cabe también destacar que gracias a que Fatio de Duillier dejó la Haya a finales de 1691 podemos tener esta importante perspectiva sobre el comienzo de la controversia por el

${ }^{44}$ Íbid., III 5, n.53: 237.

45 Íbid., III 5, n.65: 280.

${ }^{46}$ Íbid., III 5, n.69: 290.

${ }^{47}$ También es de esta opinión Pérez de Laborda (Pérez de Laborda, 1977: 170). 
cálculo, pues debido a su mudanza a Inglaterra, él y Huygens comenzaron a comunicarse por carta.

Huygens escribe a Fatio nada más recibir el artículo de Leibniz dirigido a Fatio. Aunque Huygens afirma en la carta dirigida a Leibniz el 4 de febrero de 1692 que no había enviado copia de este artículo ni ninguna parte del método de Leibniz a Fatio, lo cierto es que sí lo hizo. El 18 de diciembre de 1691 Huygens envía a Fatio algunas de las proposiciones de Leibniz, aunque es cierto que lo hace afirmando que a pesar de que el prefacio es magnífico, hay mucha oscuridad en el texto hasta tal punto que no sabe cómo llegar adecuadamente a la conclusión, e incluso añadiendo que no sabe por qué medio llegar a una de las cuadraturas. Afirma, también, que Fatio está menos versado en el cálculo y que por eso no le envía todo el método de Leibniz ${ }^{48}$.

La respuesta de Fatio es la carta del 28 de diciembre de 1691, la que según Aiton supone la semilla de la controversia, pues es donde Fatio afirma por primera vez que Newton es el primer autor del cálculo diferencial y que si Leibniz lo ha desarrollado es gracias al contacto epistolar que Leibniz y Newton mantuvieron en el pasado. Si bien es cierto que la primera mención explícita de que Leibniz ha podido plagiar el cálculo se encuentra en esta carta $^{49}$, está claro que las palabras de Fatio provienen de un contexto concreto, que es el que he señalado. Es más, es imposible conocer las motivaciones de Fatio al decir estas palabras sin conocer el trasfondo de la discusión del método inverso de tangentes. Estudiándolo con determinación, se puede

${ }^{48}$ Ch. Huygens, op. cit., 10, n.2721: 209-211.

${ }^{49}$ Íbid., 10, n.2723: 214. 
observar que las palabras de Fatio pueden estar motivadas por lo siguiente. Habiendo leído la afirmación de Huygens sobre la oscuridad que tiene el artículo escrito por Leibniz, así como la opinión de que Fatio no está lo suficientemente versado en el cálculo, él se defiende afirmando que entiende perfectamente el cálculo diferencial de Leibniz a pesar de las faltas de impresión de sus textos que están ocasionados por las faltas que tiene Leibniz, las cuales estarían realizadas a propósito ${ }^{50}$. Por lo tanto, su reacción es una defensa a una especie de desprestigio respecto a sus conocimientos, y también parece querer dejar patente su conocimiento sobre el cálculo hasta tal punto que afirma conocer de dónde proviene el cálculo leibniziano. Además, las palabras de Fatio parecen estar motivadas por una especie de venganza a Leibniz por no haber cumplido su parte del trato, pues Fatio conocía los textos de Leibniz sobre el cálculo desde hace años, así como los de Newton, pero no es hasta este preciso momento cuando acusa a Leibniz ante Huygens, mientras que antes no tuvo problema en intercambiar su método con él.

Esto podemos comprobarlo también por la vehemente insistencia que ejerce Fatio en las cartas a Huygens, quien aplaca con pocas pero decididas palabras a su antiguo asistente en la primera de sus respuestas, y quien lo vuelve a hacer en la segunda de las respuestas a Fatio, esta vez ignorando la acusación de plagio ${ }^{51}$. La primera respuesta de Huygens a la que nos referi-

\footnotetext{
${ }^{50}$ Íbid., 10, n.2723: 213.

${ }^{51}$ La segunda respuesta de Huygens, con fecha de 29 de febrero de 1692, no se encuentra en las Oeuvres Complètes de Huygens, pues los editores afirman que ésta se ha perdido (Ch. Huy-
} 
mos afirma que el mismo Newton no dice que Leibniz le haya plagiado en los Principia Mathematica, donde Newton lo nombra en el escolio del Lemma II, sección II del libro II $^{52}$, referencia a Leibniz que Newton eliminó de posteriores ediciones y que aclaraba que se habían intercambiado cartas en 1676. Fatio, sin embargo, sigue insistiendo el 15 de febrero de 1692, afirmando que si las cartas que Newton y Leibniz mantuvieron entre 1676 y 1677 se imprimiesen, dañarían grandemente la reputación del alemán. Afirma también que no puede dejar de comparar los métodos de Newton y de Leibniz, destacando la imperfección del método del último, diciendo que es una "copia estropeada" ${ }^{53}$. La respuesta de Huygens ignora las palabras de acusación, y en las siguientes cartas la discusión ha cesado.

\section{La explosión de la controversia}

Huygens había apagado las llamas de la controversia, pero el rencor de Fatio mantuvo las ascuas encendidas durante los años siguientes, e iban a volver a arder a finales de siglo ya una vez fallecido Huygens.

La controversia se reaviva cuando Johann Bernoulli propone en las Acta Eruditorum de 1696 encontrar la curva que de un punto A a un punto B y

gens, op. cit., 10, n.2745: 271). La respuesta, sin embargo, está editada en Bibliotheque universelle des sciences, belles-lettres et arts 22, 1823: 256-258.

52 Íbid., 10, n.2733: 242.

${ }^{53}$ Íbid., 10, n.2739: 258. 
solamente mediante la fuerza de la gravedad, llegue lo más rápido a $\mathrm{B}^{54}$, cuya solución es la curva braquistócrona. Las proposiciones de este tipo de retos eran habituales: tenemos otro ejemplo en el reto de la descripción matemática de la curva catenaria que el mismo Bernoulli propuso en las Acta EruditoEruditorum en mayo de 1690, donde participaron su hermano Jacob, así como Leibniz y Huygens.

Aquellos que enviaron sus soluciones al reto de la curva braquistócrona fueron Leibniz, Jacob Bernoulli y L'Hôpital. Newton, por su parte, decidió publicar su solución anónimamente en las Philosophical Transactions de la Royal Society ${ }^{55}$, aunque parece ser que Bernoulli conocía la verdadera identidad del autor. Las soluciones enviadas junto con un extracto de la solución de Newton fueron publicadas en las Acta Eruditorum de 1697.

Junto con su aportación, Leibniz añade unas palabras en las que afirma que aquellos que han encontrado una solución al problema son aquellos que él pensaba que eran capaces: Jacob y Johann Bernoulli, L’Hôpital, Huygens de estar vivo, Hudde de no haber dejado de estudiar estos asuntos y Newton si se hubiese molestado en buscar una solución ${ }^{56}$.

${ }^{54}$ J. Bernoulli, "Supplementun defectus geometriae cartesiane circa inventionem locorum", Acta Eruditorum Junio 1696: 269.

${ }^{55}$ I. Newton, "Epistola Missa ad Praenobilem Virum D.Carolum Mountague Armigerum, Scaccarii Regii apud Anglos Cancellarium, et Societatis Regiae Praesidem, in qua Solvuntur duo Problemata Mathematica a Johanne Barnoullo Mathematico Celeberrimo Proposita", Philosophical Transactions 1695 vol. 19: 384-389.

${ }^{56}$ G.W. Leibniz, "Communicatio suae pariter durarum que alienarum ad edendumfibi primun a Dn. Jo. Bernoullio...”, Acta Eruditorum mayo 1697: 203-204. 
Leibniz no nombró a Fatio como uno de los que podrían haber encontrado una solución, y éste se lo tomó como algo personal. Como reacción a las palabras de Leibniz, Fatio publica en 1699 su tratado de 24 páginas que habitualmente la bibliografía especializada sitúa como comienzo de la controversia por el origen del cálculo ${ }^{57}$. En él, además de su solución al reto de la curva braquistócrona, Fatio afirma que Newton es el primer inventor del cálculo, y señala que si Leibniz ha tomado algo prestado de Newton, es algo que deja a juicio que aquellos que hayan visto los manuscritos y cartas de Newton (como él hizo). Pero algo no menos importante es que hace referencia a las cartas que intercambió con Leibniz: afirma que si de algo pueda jactarse Leibniz no será de tenerle a Fatio como discípulo, lo cual se descubriría si alguna vez se hacen públicas las cartas con Huygens ${ }^{58}$. Esto hace referencia al fallido intercambio de métodos, y queda patente que Fatio tiene en mente, al escribir estas líneas, todo lo que ocurrió entre 1691 y 1692. A pesar de que Fatio sitúa claramente el origen de su conocimiento o sospecha de que Leibniz había plagiado a Newton, este hecho y el contexto ha sido ignorado a la hora de recapitular la controversia del cálculo.

\section{Las cartas de Fatio y Leibniz}

${ }^{57}$ F. De Duillier, op. cit.

${ }^{58}$ Íbid., 18. 
Es necesario señalar que, antes del estallido de la polémica, Fatio intentó mantener una correspondencia con Leibniz en 1694 enviándole una carta indirecta a través de Beyrie en la que se centraba en su teoría de la grave$\operatorname{dad}^{59}$. Leibniz, en su respuesta, incluye afirmaciones como que sería un placer ser instruido por Fatio en cuanto a lo que ha estudiado respecto a la atracción de los cuerpos ${ }^{60}$, lo cual muestra la disposición de Leibniz al diálogo tras la polémica a pesar de que muestra cierto desinterés en carta a Huygens ${ }^{61}$. Esta carta nunca fue respondida por Fatio, por lo que tenemos, de nuevo, un intercambio fallido entre los dos.

Ya a partir de 1699, tras la publicación del artículo de Fatio, se produce un tercer intercambio de impresiones entre él y Leibniz que confirma que el origen de la controversia se encuentra en el intercambio de métodos que mantuvieron entre 1691 y 1692. Antes de escribirle personalmente, Leibniz responde a Fatio en las Acta Eruditorum de mayo de $1700^{62}$, donde niega las acusaciones de plagio. A partir de aquí, la discusión entre Leibniz y Fatio pasa a tener lugar en cartas en lugar de en artículos, pero aunque el intercambio se torna personal, a gran escala la controversia va creciendo entre los científicos británicos y los continentales.

${ }^{59}$ G. W. Leibniz 1923ss., III 6, n.14: 44-48.

${ }^{60}$ Íbid., III 6, n.34: 85-86.

${ }^{61}$ G. W. Leibniz 2007ss., vol. 7A: 346ss; Leibniz 1923ss, III 6, n.45: 129ss.

${ }^{62}$ G.W. Leibniz, "Responsio ad Dn. Nic. Fatii Duillerii imputationes", Acta Eruditorum mayo 1700: 198-208. 
De momento, solamente una breve mención por parte de Fatio en su artículo unen el reto de la curva braquistócrona entre 1696 y 1699 con lo acaecido entre 1690 y 1692 en la correspondencia entre Leibniz y Huygens. Pero lo cierto es que cuando Fatio escribe a Leibniz como respuesta al artículo de las Acta Eruditorum de mayo de 1700, Fatio afirma que no escribe su artículo de 1699 como venganza por no haber sido enumerado entre los matemáticos posibles, como podría parecer, puesto que incluso antes habían tenido un intercambio de métodos cuyo final le pareció injusto y que Leibniz quería echarle la culpa a él y a Huygens ${ }^{63}$. Sus palabras, que niegan la venganza como motivación, parecen dar a entender precisamente lo contrario.

La respuesta de Leibniz a Fatio tuvo un tono mucho más comedido. Explica que Huygens le había propuesto el intercambio de métodos y que finalmente no funcionó. Afirma que ni en sueños había pensado que Fatio le iba a hacer daño, que tiene costumbre de alabar a aquellos que lo merecen y que su silencio respecto a las soluciones de la curva braquistócrona ha incitado a Fatio, lo cual parece indicar que la motivación de Fatio está marcada por la venganza. Además, dice Leibniz que espera que a Fatio se le recuerde por sus resultados, dejando a entender que espera que se le recuerde por sus aportaciones a las ciencias y no por estas disputas ${ }^{64}$. Como se puede comprobar, ambos señalan rápidamente el origen de sus discusiones, que se encuentra en la correspondencia que mantuvieron Leibniz y Huygens.

${ }^{63}$ G. W. Leibniz 1923ss, III 8, n.182: 463-464.

${ }^{64}$ Íbid., III 8, n.196: 504. 
Controversia sobre el cálculo infinitesimal

\section{Condusiones}

A finales del siglo XVII, aunque en los círculos académicos académicos ya circulaban dudas sobre quién fue el primero en inventar el cálculo, fue Fatio el que dio pie al origen de la controversia por la prioridad de su invención. Su motivación estaba marcada por las malas experiencias con Leibniz, las cuales comenzaron con el intercambio del método inverso de tangentes que tuvo lugar durante el desarrollo de la correspondencia Leibniz-Huygens entre 1690 y 1692, siguió con un breve intercambio de impresiones respecto a cuestiones mecánicas que Fatio decidió no continuar y culminó con el silencio de Leibniz sobre Fatio en el reto de la curva braquistócrona. Por ello, y puesto que Fatio actúa en su artículo de 1699 motivado por estas malas experiencias con Leibniz, tal y como él mismo da entender al citar el intercambio del método inverso de tangentes, se puede afirmar que ese es el origen de la controversia por la invención del cálculo.

La bibliografía especializada no ha identificado este punto como el origen de la controversia, sino que habitualmente lo ha situado en 1699 , momento en el que definitivamente estalla la controversia, como comienzo de ésta. Sin embargo, las mismas palabras de Fatio y Leibniz nos retrotraen hasta el intercambio epistolar que mantuvieron Leibniz y Huygens entre 1690 y 1692. El conocimiento del origen de la controversia y las motivaciones de Fatio de Duillier para sacar a la luz pública sus acusaciones nos muestra cómo la historia de la ciencia se encuentra lejos de estar determinada solamente por el quehacer científico. Pues siendo los científicos todos humanos, son las pasio- 
nes las que mueven sus corazones tal y como vemos en este caso, a veces para bien y a veces para mal.

Agradecimientos. Estoy muy agradecido a los profesores Juan Arana, Francisco R. Valls, Francisco Soler Gil y Jesús de Garay por animarme a hacer pública la tesis de este artículo y ayudarme con algunos aspectos técnicos. No me encuentro menos agradecido con el profesor Bartolomé Segura, cuya inestimable ayuda con el latín ha sido esencial para desarrollar este texto.

Miguel Palomo

miguelpalomo@us.es 
SECCIÓN BIBLIOGRÁFICA 
